\title{
Cytological Studies on Inflammation, Especially with Reference to the Origin of So-called Round Cells in Inflammatory Foci
}

\author{
By
}

\author{
Mizu Kojima, Mamoru Takagaki, Tsutomu Watanuki, \\ (小島瑞) (高垣衛) (綿 貫 勤) \\ Masayuki Ogata, Masabumi Kuroda and Sanae Oota \\ (緒方 諴之) \\ (黑田 政 文) \\ （太田早苗） \\ From the Pathological Laboratory of Prof. K. Akazaki \\ Faculty of Medicine, Tohoku University, Sendai
}

(Received for publication, April 18, 1958)

\section{Introduction}

The study on the origin of the mononuclear cells in inflammatory foci has made a great progress by the introduction of the technics of hematocytological examination, but on the other hand, the relation between the inflammatory cells and the blood cells seems to have come to be overestimated, leading to the advocation by some authors of theories asseverating that nearly all the cells appearing in inflammatory foci must be of hematocytic origin. These theories are indeed based on so precise cytological findings as not precedented in the past histopathological researches and have many followers among the modern researchers. In applying such theories, however, it is not to be denied that many a contradiction and inconsistency would be encountered in interpreting the various phenomena concerning inflammation. The largest cause of such foibles of these theories, I believe, lies in the neglect, slight or otherweise, of following further up the relation between the inflammatory cells and the local mesenchymal cells, especially the reticulo-endothelial cells (RE cells), and the metamorphosis of the latter cells-important points that should be discussed fully before entering upon theorising on the subject. In consideration of such negligence on these points, the present authors have undertaken a strict research, from both the angles of blood cells and tissue cells on the origin of mononuclear cells coming forth in inflammatory foci, in particular, typhoid cells in typhoid fever foci and epitheloid cells in tubercles, basing ourselves on the voluminous informations on the RES accumulated at this laboratory. As the result, we have succeeded in demonstrating that these cells, in refutation of the above mentioned theory, 
are originated, not in blood cells, but in the RE cells, a summary of our study was reported at the "7th Symposium on Hematology," 1954..1, 2),3) Since then, we have proceeded with many fundamental studies on the transformabilities of the RE cells, and arrived at many informations that serve in endorsing our assumption, at the same time proving in concrete that the above theories are nothing but a system of speculation built upon a neglect of such transformabilities of the RE cells. The details of our study were reported by Prof. K. Akazaki in a special lecture at the general meeting of the Society of Dermatology and Veneorology of Japan in May 1956.41,5) The author will report hereunder in summary on the cytological informations on the metamorphosis of the RE cells obtained at this laboratory and make some discussions - in the various phases of the inflammatory process from the side of RE cells metamorphosis, as based on these informations.

\section{State of Physiological Inflammation}

1) Phagocytes in the serous cavities. It is well known that among the mesenchymal cells, in physiological conditions, the phagocytes in the peritoneal cavity are the nearest to the mononuclear cells observable in inflammatory foci in appearance, and that the some workers ${ }^{6)}$ asserting the monocyte origin theory of these mononuclear cells have pounced upon this type of cells as the best example of wandering out into tissues and as the ground to build up their claim of monocytic origin of the mononuclear cells discovered in inflammatory foci. Murata's wide-scoped studies ${ }^{71}$ on these cells, however, has revealed that though these phagocytes in the peritoneal cavity look similar to blood monocytes they are quite different from the latter in their essential nature, and from the finding that these phagocytes are produced from the milky spots in the omentum majus formed in the embryonic stage already, they have been proved to represent local intraperitoneal phagocytes unrelated with blood monocytes. More recently, Koda has confirmed the constant existence of similar phagocytes in the thoracic cavity and the pericardial cavity and demonstrated that these are originated in the milky spots similar to those in the omentum majus and existing in the pleura and the epicardial wall. In consideration of their findings that these phagocytes are similar to the histiocytes in the subcutaneous connective tissue in their essential cytological characteristics but show also some quite dissimilar features (e.g., of being round and free, of showing nuclear lobulation and of forming neutral-red rosette), these authors hesitated to identify them with the subcutaneous histiocytes but tentatively set up a separate cell type for them under the name of the "phagocytes in the serous cavities."

2) Phagocytes in joint cavities. For elucidating the question whether 
these phagocytes in the serous cavities are in fact of different type from the subcutaneous histiocytes prossessing characteristics sufficiently specific to distinguish them from the latter or these two apparently different types represent only two different phases of the same kind of cells in metamorphosis, we chose the joint cavities as the best field of study. According to the study reported by Yamagata ${ }^{8)}$ of Fukushima Medical College, 50-350 free cells per $\mathrm{mm}^{3}$ are in permanent existence in the knee- and shoulderjoint cavities of normal rabbits and he found that about 70 per cent of these are of a type indentifiable with the phagocytes in the peritoneal cavity. Upon closer comparison of these joint cavity phagocytes with those in the abdomen, we found that while Murata's Type II cells (forming neutral-red rosette) are most prevalent in the abdominal cavity of rabbit, but his Type III cells (forming no neutral-red rosette) are more predominant in the joint cavities, where of the Type II cells only those showing no lobulation in their nuclei were conspicuous. Besides, spindle-shaped cells found only sparsely in the abdominal cavity were found relatively frequent in the joint cavities. Upon further studies directed to the synovial membrane, he found milky spots in permanent existense chiefly in its fat tissue tegumen, smaller indeed than those found in the omentum majus, and production of the joint cavity phagocytes from these milky spots. When $0.2 \mathrm{cc}$. of $10 \%$ peptone solution was introduced into a joint cavity, the cells forming such milky spots rapidly increased in number, accompanied by a corresponding increase of the phagocytes in the cavity and the number of Type II cells was found to rise therewith.

Since the joint cavities, viewed embryologically, are nothing but crevices in the connective tissues, different from the serous cavities, we are led to infer that the permanent existence of phagocytes of the same types in both these cavities means on the one hand that the phagocytes in the serous cavities are not cells of types specific to some specific locations and on the other hand that the phagocytes in the connective tissue, i.e., the histiocytes are transformable into the form of phagocytes in serous cavities. Furthermore, from the finding that the phagocytes in the joint cavities that may be looked upon as standing midways between subcutaneous histiocytes and serous cavity phagocytes come closer to resemble the serous cavity phagocytes morphologically by additional stimulation, the importance of the factors of such stimulation or activation in the process of metamorphosis of such connective tissue phagocytes is accentuated.

3) Vital staining. When rabbits are daily injected intravenously with $3.3 \mathrm{cc} / \mathrm{kg}$ of $1 \%$ trypan-blue solution for 10 days, the RE cells in the animals are stained nearly enough. By this method, we attempted to catch the process of transition of the subcutaneous histiocytes into phagocytes in the joint or the serous cavities by examination of all the RE cells 
in the whole bodies of the animals. The results showed that the spherical histiocytes in the spleen, the bone-marrows and the lymph-nodes represented such transformed cells and besides that the histiocytes in the splanchnic capsules, the bulbar conjunctiva, the lamina propria of the intestinal wall and similar tissues were far more densely packed, more polymorphic and sometimes more frequently rounded out in form than those in the subcutaneous connective tissue. Such a polymorphism of the histiocytes in many different regions is sufficient to justify the inference that the gaps between the forms shown by the histiocytes in the subcutaneous connective tissue, the joints and the serous cavities cited above are filled up hereby and that the different forms of these cells are only different phases of the same type of cells in transformation.

4) Physiological stimulation. Not to speak of the spleen and the lymph nodes, it is easy to suppose that such tissues as the bulbar conjunctiva and the propria of the intestinal wall are normally under stronger stimulations than are the subcutaneous tissues and we need not mention that the joints and the serous cavities are all constantly in the state of mechanical stimulation. It is probable that such incessant stimulations exert some decisive effects on the forms of the histiocytes in the parts concerned. For obtaining informations on this subject we conducted the following experiments:- a) When $1 \mathrm{cc}$ India ink was introduced into the abdomen of mice, carbon particles were found in the thoracic cavity and partly taken in by phagocytes there in $10 \mathrm{~min} . . \quad b)$ When $0.2 \mathrm{cc}$ of India ink was directly introduced into their thorax, in $30 \mathrm{~min}$. lumps of carbon particles were found adhering mainly on the surface of the pleura over the mediastinal cavity and some of the particles penetrated deeper into the membrane and began to be taken in by phagocytes. c) When mice were made to inhale soot for 3 hours, a proliferation of the milky spots in the pleura began soon after the treatment and in 18-48 hours accumulation of carbon particles was observed, first in the intrathoracic free phagocytes and then in the milky spots in the pleura lining the mediastinal cavity. When $0.1 \mathrm{cc}$ of India ink was introduced into the trachea of mice, similar findings were obtained in 1-48 hours. These results seem to suggest that the pleural cavity exchanges humor with the abdominal cavity and the lung, mainly through the route of the mediastinal cavity, and the intracavital free cells and the cells forming the milky spots in the pleura are constantly active in disposing of such incoming humor. Thus, the phagocytes in the thorax are under incessant stimulation and may be looked upon to remain always in a state of hyperfunction. Similar states of excitation may be easily guessed to exist in the abdominal, the pericardial and the articular cavities too (Experiments on this subject planned).

Accordingly, we may say that all the different regions and tissues in 
the body are constantly under stimulation, strong or weak, the RE cells are drawn into reactions and are activated in accordance with the kind and intensity of the stimulation to which their respective sites are subjected and they take up the different cellular forms as the concrete expression of their state of heightened function.

Now, if regard inflammation from the site of cellular response and take it to be a state where an abnormally strong mobilization of the inherent function of the cells is demanded, we may regard the serous and the joint cavities described above to be in a state of inflammation physiologically. This state we understand to fall under the appellation of physiological inflammation as proposed by Roessle ${ }^{9)}$ and emphasised by Maximow. ${ }^{10)}$ Therefore, we can easily suppose that even the subcutaneous connective tissue cells, when an abnormally high functioning is demanded, would take the form similar to or identical with the histiocytes in these cavities. Accordingly, the fact that there is a resemblance between the mononuclear cells found in inflammatory foci and the phagocytes in the serous cavities does not result from the appearance of a specific type of cells called the intra-abdominal phagocytes in such foci, as many authors have opined in the past, but indicates that both the inflammation cells and the intra-abdominal phagocytes represent morphological expressions of one and the same cellular type, that is, of the histiocytes equably in a state of hyperfunction.

\section{States of Stimulation}

When the RE cells are abnormally activated and proliferate so that the general condition of the site shows a deviation from the normal scope, we look upon such a condition as a state under stimulation. This state may occur in all the RE system in the body or may be limited to some specific organ or tissue. The former state we wish to call the general and the latter the local state of stimulation. In this meaning, the so-called inflammatory foci may be said to represent, from the view-point of the RES, a quantitatively very intense but locally circumscribed state under stimulation.

The state of general stimulation.11) The subcutaneous tissue is normally subjects to extremely weak stimulations only, so that the histiocytes here may be said to be in the form expressing a state of rest. But when we began examining the subcutaneous tissues of a number of untreated adult rabbits and mice, we found that the form and the number of the histocytes therein were rather widely different by individuals and in some they were in a state of very high activation. By systematic examination, we found that even among the untreated normal animals the level of activation and cell proliferation in the entire RES was different 
individually and the subcutaneous histiocytes were unequally activated and proliferated according to the different level of RES excitation, that is, we found some among the untreated animals were already in a state of general stimulation.

Searching for a method of experimentally inducing such a state of abnormal activation of the RES in general, we found that a single intraabdominal, inoculation of $5 \mathrm{cc} / \mathrm{kg}$ of typhoid vaccine was the simplest and surest method with rabbits. Following such a treatment, the histiocytes were found to undergo manifold transformation and proliferations. Since no emigration of leucocytes occur throughout, we could follow up the transformation processes of the histiocytes without being troubled by the interference of leucocytes. The degree of transformation of the subcutaneous histiocytes following inoculation of typhoid vaccine is markedly affected by the original level of RES activation of the animal, but in a rabbit apparently in relative normal health, the following progress seems to be the usual course. After the inoculation, the nuclei and the cell bodies of the histiocytes in existence begin to diminish in size and the focal arrangement of these cells becomes conspicuous. Soon, the condensed histiocytes showing such an arrangement begin to separate into solitary free cells and become gradually spherical, while many vacuoles appear in the cell bodies, which soon disappear and on the 3rd or 4th day a picture of degeneration comes to the foreground. A perceptible proliferation of hitiocytes is usually observed on the 5 th or 6th day. Before the reappearance of such histiocytes, large-sized cells of variable but predominantly spindle-like form comes into conspicuous appearance which increase gradually in number by active mitosis. These cells have large round or oval nuclei and their bodies are ample, often being linked together as if in mutual syncytium. The mitochondria of these cells are of short threador rod-form and are scattered throughout the cell bodies, but gradually take on short rod-like or granular form and gather densely around the nuclei and when minute neutral-red granules come to increase in number and mix with the former granules, the extracellular part of cytoplasma falls into vacuolar degeneration, soon the cell body is cut off from the vacuolated part and form a single cell and then is converted into young fixed histiocytes by subsequent contraction. The young fixed histiocytes thus formed are then proliferated by division (chiefly amitosis), resulting in focal or fascicular formation of proliferated histiocytes. We gave the name of prehistiocytes to such cells representing the preliminary stage of histiocytes. Those cells almost totally lack in phagocytosis, are scarce and hard to define in normal subcutaneous tissue of rabbit, but increase enormously in the state of stimulation and become the main factor of histiocytic proliferation. The multifariousness in form of the cells coming 
forth in the course of such proliferation and the manifold combination of the transformation processes of these cells into histiocytes calls the multiform hitiocytes into existence. The small-sized histiocytes appearing under stimulation represent one of the types of such multifarious new-born histiocytes and comprise many kinds including those directly transformed from prehistiocytes on the one end and those formed by division of histiocytes on the other, showing diverse forms according to their genesis. In the former type, the cells are rich in mitochondria, are predominantly free and are often negative in vital staining. The latter have bodies filled with neutral-red granules, are closely similar to the stable large-size histiocytes in appearance and show active phagocytosis. From these observations, we may summarize our cytological findings on the subcutaneous tissue under stimulation into the following major items:-a) Increase of the cells in number. b) Proliferation of prehistiocytes. c) Growth of histiocytic nidal or fascicular proliferation foci. d) Generation of numerous small-sized round free histiocytes. From the observed fact that the occurrance and the intensity of these major findings show approximate parallelism to the level of activation and proliferation in the entire RES, we may say these findings offer concrete means for estimating the general state of

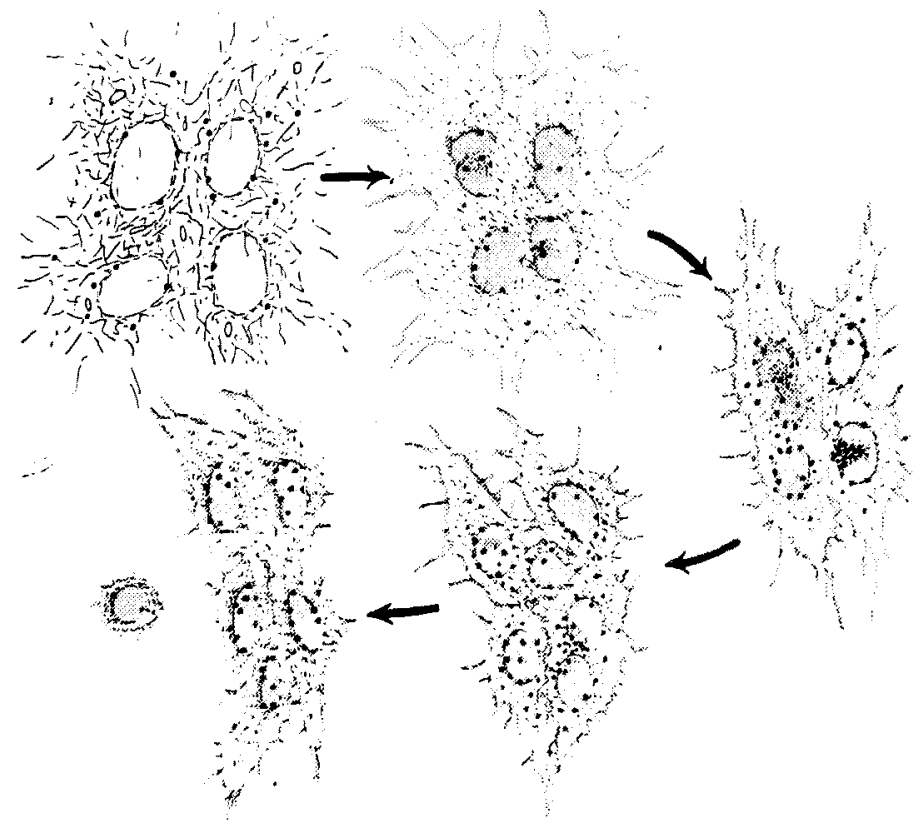

Fig. 1. Schematic representations of inversion of prehistiocytes into spindle-shaped and rounded histiocytes. Supravital staining with neutral-red and Janus-green. 


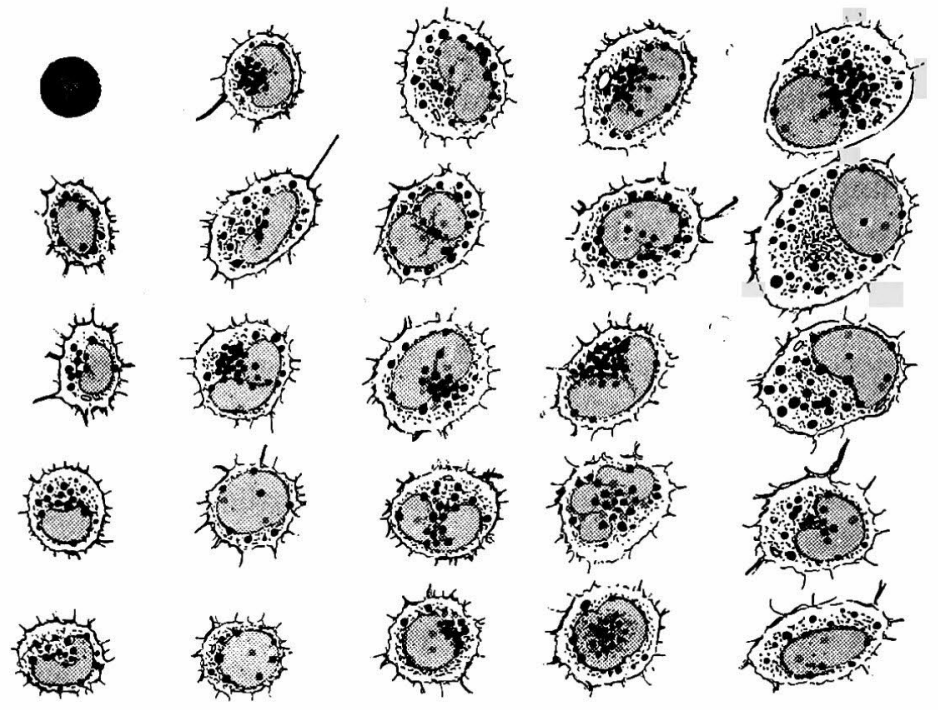

Fig. 2. Free rounded histiocytes from the subcutaneous connecture tissue of a rabbit injected intraperitoneally with typhoid vaccine. Supravital staining with neutral-red and Janus-green. The black corpuscle at upper left side shows a red cell.

RES activation.

IV. Vicissitudes of the Histocytes in Inflammation Foci (Localized Stimmulated Condition)

The phlogogenic agents used in our experiments consisted in the animal's own blood, $1 \%$ cholesterin added to a Tween 80-physiologic saline solution, $1 \%$ agar solution, silica dust, bone powder, typhoid vaccine, horse serum, BGG suspension, lepromin etc.. These agents call forth more of less characteristic responces respectively, but the inflammatory reactions caused by introduction of these substances have the common feature of being free of undue interference of leucocytes, so that the transformation process in the histiocytic cell reaction can be clearly grasped. We will refrain here from entering upon the details of the individual inflammation processes, and describe only the fundamental type of the transformation of the histiocytes coming into existence during the process.

The first change to be observed is the initial reaction coming forth just after the introduction of the inflammatory agent down to the occurrence of leucocyte infiltration. Immediately after the introduction, the subcutaneous tissue begins to swell and becomes edematous, while most of the existing histiocytes are reduced in size. The cell nuclei are pycnotized, becoming round or of elongated-ellipsoid form. The cell bodies are also diminished in size, become spherical, spheroidal or of 
long spindle-form and filled with coarse neutral-red granules, while numerous unstainable vacuoles also make appearance. Soon the round free cells begin to increase, the cord-wise arrangement at first prominent becomes cut up and the cells become solitary and free. Meanwhile the fibrocytes also mostly fall into degeneration and come to be replaced by large-sized, slightly more basophil cells with elliptical nuclei rich in chromatin. Leucocyte infiltration comes in usually at this stage, that is, 1-3 hours after the introduction of the inflammatory agent. The infiltration of granulocytes reaches the maximum intensity in 6-12 hours, then falls off gradually, while the picture of degeneration becomes frankly distinct, and the leucocyte infiltration fades out from the inflammation focus on the 2nd or 3rd day. The emergence of blood monocytes was usually slight in the inflammation foci induced by the above-mentioned agents. These monocytes come out together with the granulocytes and mixed with them in the inflammation foci, and take in the latter in phagocytosis but disappear from the foci following their extinction. The histiocytes in such foci of leucocyte infiltration have their bodies swollen up and mostly die away, but in the sites at some distance from the center of leucocyte infiltration, the cell proliferation process similar to that in the state of stimulation cited above in form but far more vehement in degree is rapidly developed. A prominent focal proliferation begins, releasing round or spheroidal cells, mainly consisting in monocytoid cells, while the chromatin in the larger-sized fusiform cells are increased, both those size and form become more various and those cell bodies become more basophil. These large-sized cells are incessantly changed over into medium- or small-sized cells while they proliferate rapidly themselves, so that the focal cells come to comprise more and more younger cells. The invasion of such cells into the central part of the foci depends on the level of devastation of the foci. In the cases of small and mild foci, highly basophil cells of mainly spindleform penetrate into the necrobiotic foci while undergoing rapid proliferation and utilizing the remnant collagenous fibres and fibrine masses as footholds. The nuclei of these cells are rich in chromatin, frequently contain nucleoli and many figures of nuclear division are also observed. The round cells of varying sizes born in the course of cell proliferation infiltrate into the liquid past devoid of trabecular tissues. These free cells in rare cases are observed to undergo mitosis, but in most cases the new cells seem to proliferate further through amitosis, and in conformity with this observation we found many nuclei in indefinite lobulation. The foci mainly containing large-sized fusiform cells mixed with large and small round cells then make transition into foci mainly filled with small-sized round cells. In the case when the former stage persists for 3-4 days, this transition occurs on the 4th-6th day. These free cells are similar to lym- 
phocytes, monocytes or plasmocytes in appearance, but are dissimilar from any of them in that they show active phagocytosis. As the liquid content in the focus is decreased, the number of the small-sized round cells is also reduced, while the medium-sized formed under the same mechanism as the former become predominant. The bodies of such cells gradually lose their characteristics of free cells, the existence of matters taken in by phagocytosis become rarer to observe and the circumscription of the focus becomes more marked. The circumscribed focus remains a while in the stage of predominance of medium- and small-sized cells, but in other sites the free cells become very rare and the nuclei of the large-sized spindleform basophil cells hitherto in predominance become uniformly round and medium-sized and lose in their chromatin contents. Their cell bodies become multipolar and more acidophil, and their mutual boundaries become blurred. These-after, the degeneration of these cells becomes more prominent, their number falls off rapidly and slowly the normal tissue is restored. In the interim, the findings observed in the subcutaneous tissues under general stimulation above is retained over a long period. The remnant localized foci sometimes show the structure resembling milky spots, wherein also the predominance of small-sized round cells is gradually usurped by multiform cells, the picture of degeneration becomes more prominent and belatedly the normal state is reinstated here too. The above-cited free round cells are modified into different forms by application of different agents. E.g., when cholesterine solution was administered, vesicular cells were found in predominance, after BCG suspension, lymphocytic cells of varying size were most frequent and after Mitsuda's reagent solution for leprosy, plasmocytoid cells were most numerous in the acme stage.

The above-described vicissitudes in the RES response in inflammatory foci may be schematically represented as in Fig. 4. In the uppermost line is indicated the intensity of the stimulation, where $\mathrm{N}$ means normalcy and $\mathrm{R}$ the stimulation. The phagocytic histiocytes are shown above and the prehistiocytes below the horizontal medianline. The existence of prehistiocytes is not clear in normal state, fixed-type histiocytes being predominant and nearly no free round cells being observable. As the intensity of the stimulation increases, the prehistiocytes come into more pronounced existence and they become more active. At the same time, histiocytes are actively produced, of which the free round-type cells gradually rise to majority in number. When the stage of maximum stimulation $(\mathrm{R}+\mathrm{H})$ is attained, the most activated and young prehistiocytes become highly predominant and the free cells also rise to the maximum in number (In actuality, the maximum predominance of the free round cells comes a little later than that of the prehistiocytes). With the abating of the in- 


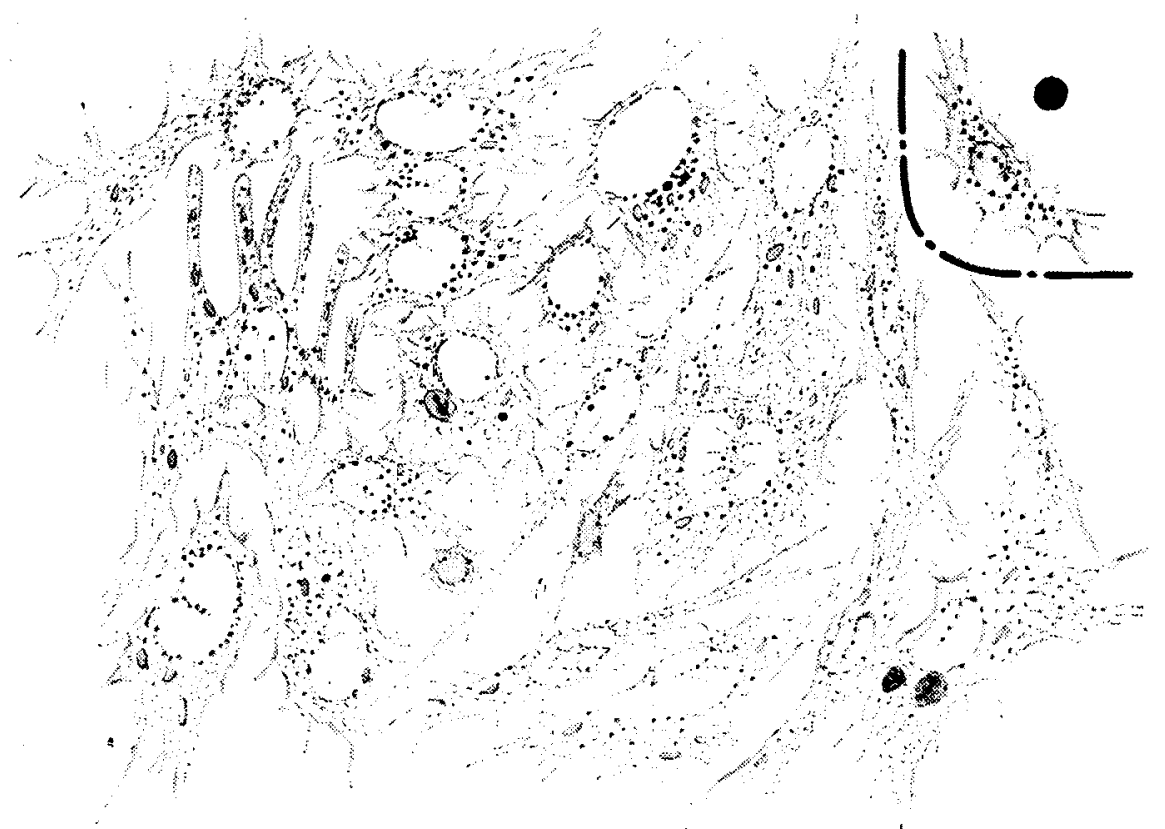

Fig. 3. Prehistiocytes from the inflammatory foci caused in the subcutaneous tissue of a rabbit. New-born histiocyte in upper right side. Supravital staining with neutral-red and Janus-green.

flammatory stimulation, the activation and rejuvenation of the prehistiocytes also gradually fall off, their number decreases, and the number of free round cells also decrease in parallel, the fixed-type cells are augmented and the normal state is approached. Then these fixed-type cells too are reduced in number, while natured cells gradually take the load and finally the normalcy is restored. The above summary has sketched the temporal variation of the metamorphoses in RES accompanying the progress of inflammation, but the same schema is applicable in studying differences in the cytological findings in different positions of the inflammatory sites subjected to a different degree of RES stimulation. So, let the condition in the stage of $\mathrm{RH}$ stand for that in the focal center and $\mathrm{N} \pm$ for the sites at a distance too large for the influence of the inflammatory stimulation to reach, the state of cellular reaction in the sites, near to and far from the focal center between there two positions can be read in the schema. Of couse the breadth and the rise and fall of the curve should be different with different intensity of the stimulation applied. Besides, we must point out that the data indicated in this schema are applicable to the cases of physiological inflammation as well. Namely, we can adapt the state under $\mathrm{N} \pm$ to that in the normal subcutaneous tissue and then the forms 
of histiocytes given under $\mathrm{R} \#$ to those in the abdominal and the pleural cavities and in the spleen, those under $\mathrm{R}+$ to the phagocytes in the joint cavities and identify the state either of $\mathrm{R}+$ or $\mathrm{R}+$ with that in the propria of the intestinal mucosa, the conjunctiva or the splanchnic capsules. In the light of such observations, we can readily understand the morphological similitude of the round cells in the inflammation foci and the phagocytes in the serous cavities cited above.

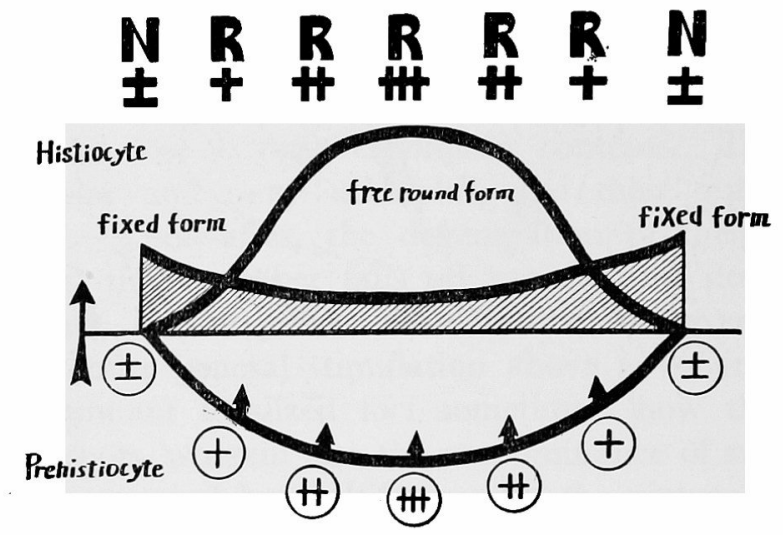

Fig. 4. Variation of the formation of histiocytes in inflammatory foci.

\section{Relation between Histiocytes and Other Cell Types}

As described above, the histiocytes go through rather kaleidoscopic changes in inflammatory foci following the progress of inflammation, so the discrimination between the histiocytes and cells of other origins naturally becomes an important operation.

1) Histiocytes versus monocytes. We have reported on the vicissitudes of monocytes in inflammatory foci on various occasions, and in our present experiments, we could confirm that the monocytes coming forth subsequent to the infiltration of granulocytes are capable of considerable phagocytosis, and at the same time we could reaffirm our original contention that these monocytes do not further divide and proliferate but always eventually degenerate and disappear. On the other hand, the monocytoid transformation of the histiocytes runs different courses in different stages. First, the small lobulated cells formed prior to the emergence of the granulocytes and the cells of the same type released from the nidal proliferation of surrounding tissue at the stage of appearance of granulocytes have their bodies filled with granulocytic granules and thus show an appearance dangerously similar to the monocytes filled with similar granules. The new-born prehistiocytes produced in the vortex of granulocyte 
Fig. 5

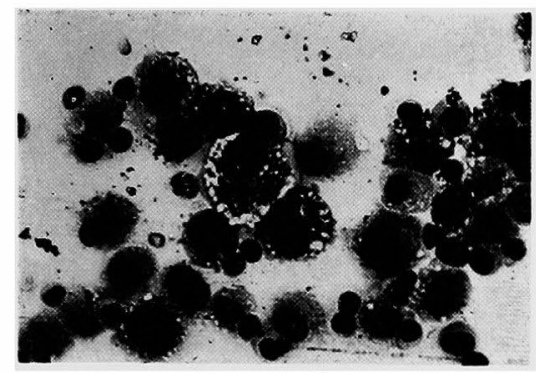

Fig. 6

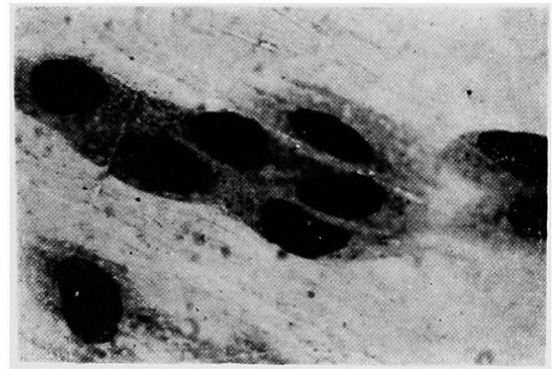

Fig. 7

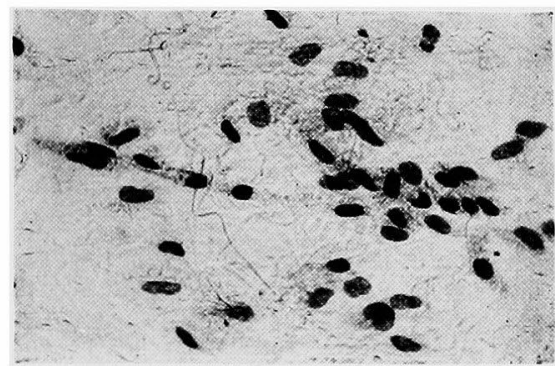

Fig. 8

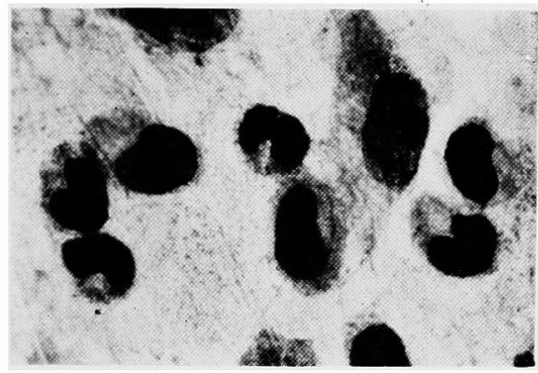

Fig. 5. Remarkable carbon-containing macrophages from pleural cavity of a mouse 5 days after inhalation of lamp-soot for 3 hours. Giemsa stain.

Fig. 6. Strech preparation of the subcutaneous tissue of the non-treated rabbit. Focal arrangement of histiocytes. Giemsa stain.

Fig. 7. Strech preparation of the subcutaneous tissue of a rabbit 24 hours after injection of $10 \mathrm{cc}$ of typhoid vaccine intraperitoneously. Focal and cordal arrangement of histiocytes and appearance of new-born histiocytes and prehistiocytes in the focal arrangement. Giemsa stain.

Fig. 8. New-born histiocytes having kidney-shaped nucleus similar to monocytes. From subcutaneous tissue of a rabbit 2 days after local injection of Mitsuda's lepromin reagent, which was previously injected with promin intravenously. Giemsa stain.

infiltration display active phagocytosis without delay. The nuclei of such cells preferentially show various kinds of lobulation successively. Their chromatin nets are delicate and fine, their bodies contain much matter taken in phagocytotically and always show more or less advanced degeneration. These also look closely resembling monocytes under Giemsa's staining, though larger in size. The cells of the two types above are observed in large number in the stage of declining granulocytes in ordinary inflammation cases, and the coincidense of this stage with that of the acme of the monocyte level curve may be especially noteworthy. We have succeeded, as reported below, in expediting or retarding the onset of such a stage of monocytoid cells reaction by previously activating or depressing the function of the RE cells, and such results also convinced us of the 
Fig. 9

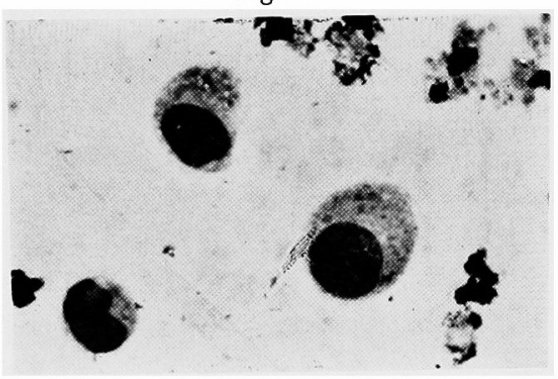

Fig. 10

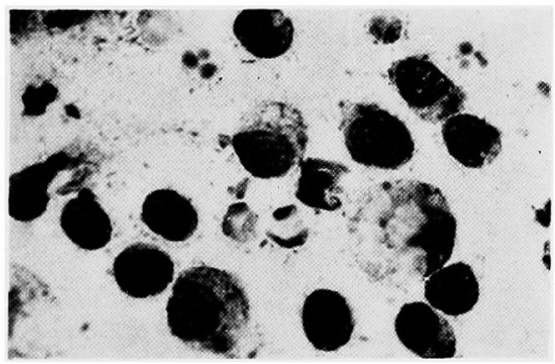

Fig. 11

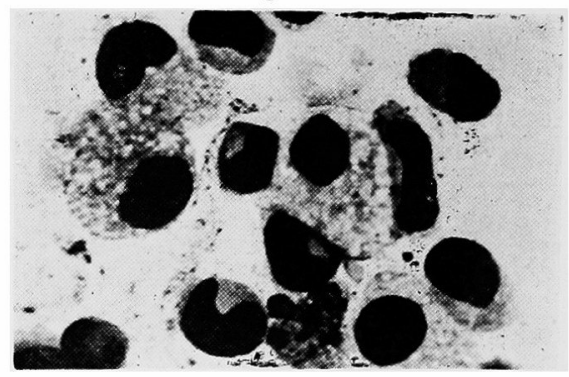

Fig. 12

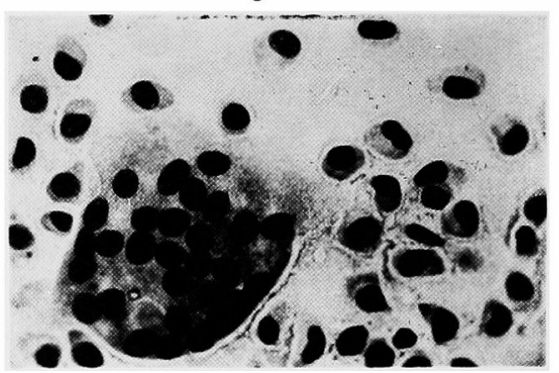

Fig. 9. Appearance of two histiocytes similar to plasma cells and a monocyte-like histiocyte. From subcutaneous tissue of a rabbit 5 days after local injection of $1 \%$ aquous solution of agar. Giemsa stain.

Fig. 10. Accumulation of new-born histiocytes similar to lymphocytes. From the cover glass 2 days after inserted into the subcutaneous tissue of a rabbit, which has been previously injected with cardiolipin-lecithin intravenously. Giemsa stain.

Fig. 11. Foam cells showing transitional varieties among surrounding hystiocytes similar to monocytes or lymphocytes. From the cover glass 2 days after inserted into the subcutaneous tissue of a rabbit, which was previously injected with cholesterin intraperitoneally. Giemsa stain.

Fig. 12. Giant cell formation on a cover glass after 4 days inserted into the subcutaneous tissue of a rabbit, which was previously injected with promin intravenously. Giemsa stain.

histiocytic origin of these cell types. The monocytoid cells released from the above prehistiocytes in the stage of further activation where they have turned into cells with gigantic and coarse nuclei and strongly basophil bodies, are usually small-sized cellules with pycnotized bilobular eccentricstanding nuclei, rich in mitochondria and frequently show neutral-red rosette formation. These cells usually come forth prior to the lymphocytoid or plasmocytoid histiocytes described below and their lobulated nuclei seem to suggest the amitosis. The appearance of their cytoplasma supravital staining coincided with that of lymphocyte-type or plasmocytoid histiocytes. They show generally feeble phagocytic ability. 
In the above, we have described in summary three types of monocytoidized histiocytes, their different types are due to the difference in the characteristics of the prehistiocytes in different stages. These cells sometimes show the appearance of being different in kind from the fixed histiocyte in the vicinity, due to secondary migration and aggregation, but a nearer study of the specimens will give multiple evidence of the release and production of these from the prehistiocytes.

2) Histiocytes versus lymphocytes and plasmocyte. The rounded histiocytes showing some or all the features of eccentric-standing nuclei rich in chromatin, highly basophil cell bodies and clear field around the nuclei would look closely resembling plasmocytes, but the conspicuous phagocytic ability denies the possibility of these type of histiocytes changing over into plasmocytes proper. Besides, the more or less variable size of these cells, the gray tint seen under May-Giemsa's staining in the basophil bodies, the prominent nuclear membrane, the fine nuclear nets, the frequent transition of the nuclear form to the oval, the rosette formation by minute neutralred granules under supravital staining, the enormous number of mitochondria found in the ample cell bodies, of these cells are findings essentially dissimilar from the findings obtained from the plasma cells discovered simultaneously in many sites of the same body. Secondary transformation of such plasmocytoid histiocytes (vesicular cells, epitheloid cells etc.) seem to characterize some kinds of foci.

Similarly, the lymphocytoid cells are identical in form with the phagocytes Type I in the joint cavities, can pass over into either plasmocytoid or monocytoid histiocytes and it is difficult to discriminate between these three types of cells. Cells of these three types probable constitute the essential elements consisting the round-cell infiltration or the so-called lymphocytic infiltration in inflammation foci.

3) Histiocytes versus giant cells. We have already elucidated in our studies on tuberculous foci and experimental subcutaneous inflammation induced by introduction of bone powder ${ }^{12}$ ) that the multinuclear giant cells observed in inflammation foci are derived from the RE cells and pointed out the errors bidden in the results of Forkner and Ikuta's studies, hitherto cited as the mainstay of the monocytic origin theory of these cells, so that we are convinced a finis has been placed on the problem of the genesis of these cells. In the present study, we have mainly proceeded with researches on the histogenetic development of giant cells. The results in summary showed that the formation of giant cells may be interpreted in a row with the focal formation of histiocytes in these proliferation process and may be attributed to a miscarriage in the course of their proliferation process. Under stimulation, we have found a few syncytic cells consisting mainly of prehistiocytes, but the stage of highest production of giant cells 
is the stage where the highly basophil spindle-form cells are actively proliferating and at the same time releasing round cells of varied size in full activity. In this stage, when the active proliferation of cell nuclei overreaches the speed of cell division, the giant cells can be produced. Consequently, the nuclei of the giant cells thus produced are identical in nature with the nuclei in the round mononuclear cells existing in the vicinity, their cell bodies are also highly basophil, often contain similar neutralred rosettes, though larger in size and similarly contain very abundant mitochondria.

Even the histiocytes once turned round and free may in some cases become polynuclear cells by nuclear division, but in such cases, the number of such nuclei is limited below 5 or 6 and their cell membran is more definite than in the cases of the above giant cells. The new-born giant cells go on increasing in the number of nuclei for a short time after their production, but soon their nuclei become swollen, the amount of chromatin decrease, unstainable vacuoles begin to appear in their cytoplasm and their mitochondrias diminish in quantity paralleling the reduction of their basophilie and the alterative findings become conspicuous, then before long these giant cells also finally disappear from the inflammation focus as do all the other free round cells. The phagocytic capacity of these giant cells shows a parallelism with the youngness or maturity at the time of their formation and in the time thereafter.

\section{Conclusion}

1) The RE cells in the living organism are more or less activated and show a variety of metamorphosis according to the functional demands made upon them in their respective sites. Thus, the morphological dissimilarity between the histiocytes in the serous cavities and the subcutaneous tissues represents only a difference in the phases of such metamorphosis, the form of the former being due only to the higher stimulation to which the serous cavities are constantly subjected to. Consequently, when the histiocytes in the subcutaneous connective tissue are under stimulation of a comparable intensity, these will also take upon a form resembling those in the serous cavities. Accordingly, we believe it is a mistake to presume that the similarity of the mononuclear cells observed in the inflammation foci to the phagocytes in the serous cavities leads to the interpretation that some phagocytes from the serous cavities-a specific cell type- or blood monocytes have come into the foci, as many have opined in the past, but in reality these represent nothing but a specific form of cells of one land the same type under stimulation. The state where the histiocytes show an inflammatory form in normal conditions too, as do the phagocytes in 
the serous cavities, we understand to fall under the idea of " physiological inflammation" as proposed by Rössle and Maximow.

2) The RE cells are thus activated to extents variable with their respective locations, but there is of course a natural limit of normalcy to such an activation. The state when such a normal limit is surpassed we have called the state under stimulation. Having induced a state under stimulation experimentally and studied the response of the entire RES in such a state, we found that we can approximately estimate the level to which the entire RES is activated by examining the metamorphosis picture of the subcutaneous histiocytes. At the same time, through cytological examination of the metamorphosis of the subcutaneous histiocytes in such a state, we succeeded in ascertaining the fundamental processes of the multiple metamorphosis, such as the transformation of the subcutaneous histiocytes into round and free cellules, occurring in the inflammation foci, without being troubled by the interference of blood cells. During the experiments, we noted the existence of prehistiocytes serving as the sources whence the histiocytes are proliferated. In summary of our outstanding findings concerning the subcutaneous tissues under stimulation, we can point out the increase of prehistiocytes in number, the focal proliferation of the histiocytes seemingly due to the local proliferation and metamorphosis of such prehistiocytes and the increase of small-sized round histiocytes derived from the pre-existing histiocytes or directly from prehistiocytes.

3) From such a view-point, we may interpret the inflammation induced in the subcutaneous tissues by introduction of phlogogenic agents to represent a state of localized but highly intense stimulation. In such a case, the apparent course of transformation of the local histiocytes is essentially indentical with that in the state of general stimulation described above, but far more intense in degree. Here, the activation and proliferation process of the prehistiocytes becomes much stronger, both quantitatively and qualitatively, their transformation into histiocytes goes on much more actively and quickly, and the histiocytes produced consist mainly in round free cells of varying size, fixed-type cells being sparse among them. With the abatement of the stimulation, however, the level of prehistiocyte activation also falls off and the conversion into fixed-type histiocytes comes gradually into the fore-ground.

4) The round and freed histiocytes outwardly resemble the monotyes, the lymphocytes and the plasmocytes rather closely, but on nearer examination, are found to be not identical with any of these cells, so that we are not inclined to support the theories postulating the transition of the RE cells into such cell types. The formation of giant cells is attributable to a miscarriage in the course of focal proliferation of such histiocytes, and the chance of such misformation is large in the stage when the pre- 
histiocytes are in active proliferation and also when the small-sized round cellules are rapidly produced. The giant cells thus produced follow the same fate as the free round cells and disappear before long.

\section{References}

1) Hasegawa, H., Acta haem. Jap., 1954, 17, 433.

2) Uekane, K., ibid., 1955, 18, 107.

3) Akazaki, K. \& Kojima, M., 1954, 7th Symposium on hematology, 121.

4) Akazaki, K., Hiseishi (Jap.), 1956, 66, 376.

5) Akazaki, K. \& Kojima, M., Nihon-Rinsho (Jap.), 1957, 15, 5.

6) Amano, S., Ketsuekigaku-no-kiso (Jap.), Vol. 1, Tokyo, 1949.

7) Murata, J., Acta haem. Jap., 1955, 18, 38.

8) Yamagata, Y., Tr. Soc. Path. Jap., 1956, 45, 297.

9) Rössle, R., Verh. dtsch. path. Ges., 1923, 19, 18.

10) Maximow, A., Arch. Path., 1927, 4, 557.

11) Kojima, M. \& Watanuki, T., Tr. Soc. Path. Jap., 1956, 45, 389.

12) Ogata, M., ibid., 1956, 45, 390. 